\title{
Le gaz naturel du bassin du Levant et Chypre : bienfait ou problème?
}

The natural gas of the Levantine Basin and Cyprus: benefit or problem for the island?

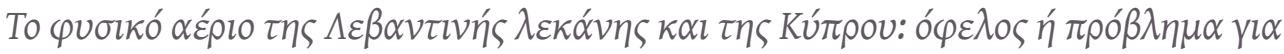

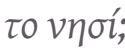

Joëlle Dalègre

\section{CpenEdition}

\section{Journals}

Édition électronique

URL : https://journals.openedition.org/ceb/14679

DOI : $10.4000 /$ ceb. 14679

ISSN : 2261-4184

Éditeur

INALCO

Édition imprimée

ISBN : 9782858313341

ISSN : 0290-7402

Référence électronique

Joëlle Dalègre, « Le gaz naturel du bassin du Levant et Chypre : bienfait ou problème ? », Cahiers

balkaniques [En ligne], 46 | 2020, mis en ligne le 25 février 2020, consulté le 06 juillet 2021. URL : http:// journals.openedition.org/ceb/14679; DOI : https://doi.org/10.4000/ceb.14679

\section{(c) (7) (8)}

Cahiers balkaniques est mis à disposition selon les termes de la Licence Creative Commons Attribution - Pas d'Utilisation Commerciale 4.0 International. 


\title{
Le gaz naturel du bassin du Levant et Chypre : bienfait ou problème?
}

\author{
The natural gas of the Levantine Basin and Cyprus: \\ benefit or problem for the island?

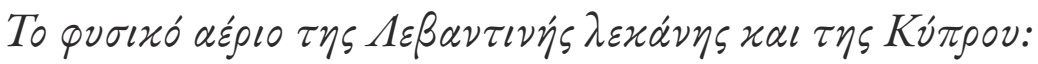

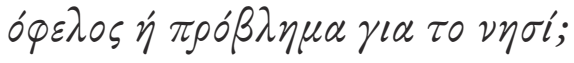

Joëlle Dalègre

CREE-Inalco

La découverte en Méditerranée orientale d'un bassin d'hydrocarbures qui pourrait égaler celui de la mer du Nord a provoqué l'enthousiasme des États riverains qui espéraient à la fois atteindre l'autonomie énergétique et faire de lucratives exportations. Mais, il leur faut réunir des conditions géologiques favorables, des possibilités techniques et commerciales d'exportation et une stabilité politique qui encourage des investissements à long terme. Autant dire que dans le bassin $d u$ Levant, entre Chypre, la Turquie, la côte moyen-orientale (Syrie, Liban, Israël, Gaza) et l'Égypte, cette dernière condition pose de sérieux problèmes. Cet article se concentre sur l'île de Chypre: dispose-t-elle de gisements importants ? Dans quelles conditions peuvent-ils être exploités ? Le gaz naturel sera-t-il pour l'île un don du ciel ou une difficulté supplémentaire ?

\section{Les ressources}

À la fin des années 1960, des sociétés israéliennes ont effectué des sondages pour trouver des hydrocarbures en Méditerranée, mais sans succès décisifs. Les premiers gisements gaziers découverts en 1999 et 2000 dans les eaux israéliennes sont Noa et Mari-B; en décembre 2003, Shell détecte d'importantes ressources off-shore au large du delta du Nil et, dès lors, les choses s'accélèrent. En janvier 2009, le 
consortium américano-israélien Noble Energy (Texas) Isramco-Delek-Avner-Dor repère le site de Tamar, puis Dalit 1, dans les eaux israéliennes; suivent, entre 2009 et 2013, Dolphin, Shimson, Tanin, Karish, mais surtout, en 2010, l'important gisement de Leviathan à $130 \mathrm{~km}$ à l'ouest de Haïf, dont les réserves sont estimées à 450 milliards de mètres cubes ${ }^{1}$. Les estimations de l'US Geological Survey publiées en $2010^{2}$ - des réserves allant jusqu'à 3,45 trillions de mètres cubes de gaz naturel dans l'ensemble du bassin - stimulent les recherches, et d'autres prospections sont faites au large de Gaza, dans les eaux du Liban et de la Syrie.

En 2006, la République de Chypre (de facto dirigée par les Chypriotes grecs qui, depuis 1974, gèrent les deux tiers de l'île non occupés par les Turcs), seul État chypriote internationalement reconnu et membre de l'Union européenne, décide une première campagne de recherches. Elle fixe sa zone économique exclusive (ZEE), y dessine treize blocs, et lance en 2007 un premier appel d'offres concernant onze des treize blocs. Finalement seul Noble Energy-Delek obtient un permis dans le bloc 12; en 2012, il annonce la découverte du gisement Aphrodite, à $34 \mathrm{~km}$ à l'ouest de Leviathan. Une seconde vague d'appels d'offres a lieu en 2012, le bloc 12 reste à Noble Energy-Delek, les blocs 10 et 11 vont au français Total et les blocs 2, 3 et 9 à l'ENI-KOGAS (italien et sud-coréen); l'ENI obtient également au troisième tour les blocs 6 et 8. Les espoirs sont grands à Chypre : les années 2012-2013 sont celles de la crise financière, une découverte rapide viendrait donner une garantie de poids au gouvernement pour obtenir de l'Union européenne des conditions meilleures. Hélas, après un certain nombre de désillusions, aucun nouvel endroit vraiment riche n'est repéré ${ }^{3}$. Mais la découverte dans les eaux égyptiennes du gisement dit Zohr, en 2015, redonne de l'espoir. En effet, Zohr est de loin le site le plus riche, des possibilités de 850 milliards de mètres cubes, à $6 \mathrm{~km}$ de la limite entre les ZEE chypriotes et égyptiennes. En novembre 2015, British Gaz Petroleum (BGP) rachète les parts de Noble dans le bloc 12, et, en 2016, Chypre lance un troisième appel d'offres: Exxon-Mobil uni à Qatar Petroleum obtient le bloc 10, voisin de Zohr, où il estime que les conditions géologiques sont prometteuses ${ }^{4}$... L'ENI obtient le bloc 8, et ENI-Total, le bloc 6. C'est là que l'ENI annonce au début de février 2018 la découverte d'un gisement Calypso,

1. On peut trouver des chiffres différents car ils sont estimés en billions et trillions de pieds cubes (d'où des problèmes de conversion) et restent des estimations qui, au fil du temps, sont revues à la hausse ou à la baisse; s'ajoute la différence entre les réserves et les réserves exploitables.

2. USGS, 2010.

3. Reuters, 26/3/2015.

4. STUDER, 2017. 
comparable à Zohr ${ }^{5}$. En février 2019, Exxon-Mobil annonce une découverte au large de l'île qui pourrait représenter entre 140 et 230 milliards de mètres cubes de gaz naturel ${ }^{6}$. Au début de juillet 2019, la République de Chypre accorde de nouvelles autorisations de forage et en renouvelle d'autres sur les blocs 2, 3, 6, 8, 9, 10, 11, 12 que se partagent Total-ENI, Exxon-Mobil-Qatar Petroleum, ENI-KOGAS et Shell-Noble. Le nombre de candidats prouve que les ressources en gaz naturel sont certaines; la géologie laisse encore espérer, mais l'exploitation se mêle très vite à la question du partage entre États et du droit de la mer.

\section{Le droit de la mer et la dispute autour de la zone économique exclusive}

En 1982 a été signée la Convention des Nations unies sur le droit de la mer dite de Montego Bay qui est entrée en vigueur en 1994. Lors de sa création, quatre États s'y sont opposés, les États-Unis, Israël, la Turquie et le Venezuela. Depuis lors, le texte a été signé par 168 pays (en 2016), 17 ne l'ont pas encore fait, dont la Turquie et Israël, 15 l'ont signé, mais pas ratifié, dont les États-Unis et la Libye. Ses articles prévoient, entre autres, la possibilité pour les États riverains d'étendre leurs eaux territoriales jusqu'à 12 milles nautiques $(\mathrm{MN})$ et de se réserver une zone économique exclusive jusqu'à $200 \mathrm{MN}$ (articles 55 à 75). Dans cette zone, l'État reçoit (art. 56):

[...] Les droits souverains aux fins d'exploration et d'exploitation, de conservation et de gestion des ressources naturelles, biologiques ou non biologiques, des eaux surjacentes aux fonds marins, des fonds marins et de leur sous-sol, ainsi qu'en ce qui concerne d'autres activités tendant à l'exploration et à l'exploitation de la zone à des fins économiques, telles que la production d'énergie.

En cas d'espace disponible inférieur à $400 \mathrm{MN}$, les États concernés doivent s'en tenir à la ligne médiane entre eux.

Le texte définit également les droits du plateau continental (le prolongement submergé du continent), mais en des termes plus flous. En termes juridiques, ce plateau s'arrête à $200 \mathrm{MN}$ comme la ZEE, mais on tient compte aussi de la définition géologique, car - c'est rare, mais possible - ce plateau peut s'étendre au-delà des $200 \mathrm{MN}$. Dans ce cas, il peut aller légalement jusqu'à $350 \mathrm{MN}$; or l'État concerné reçoit sur lui les droits d'exploration et d'exploitation des ressources du fond marin et du sous-sol, des droits comparables à ceux de la ZEE.
5. Boursedirect, $8 / 2 / 2018$.
6. Exxon-Mobil, 1/3/2019. 
212 Politique et sociétés à Chypre aujourd'hui

L'État côtier doit déposer un dossier auprès de l'ONU pour indiquer les limites de sa ZEE et celles du plateau continental qu'il souhaite fixer. La Turquie et Israël n’ont pas signé la convention, la Grèce et la Turquie depuis 1973 sont en conflit sur la définition de leurs eaux territoriales, les rapports entre Israël et ses voisins sont connus, la définition des ZEE ne peut donc qu'être conflictuelle.

La République de Chypre porte ses eaux territoriales à $12 \mathrm{MN}$ en 1964 (validé par l'ONU en 1993), elle adopte en 1974 une loi sur son plateau continental et, en 2004, fixe sa ZEE aux 200 MN. La même année, elle signe un accord avec l'Égypte qui délimite leurs ZEE respectives, en 2007, un accord avec le Liban, et en décembre 2010, un accord avec Israël. La difficulté présente naît donc de ses rapports avec la Turquie. 


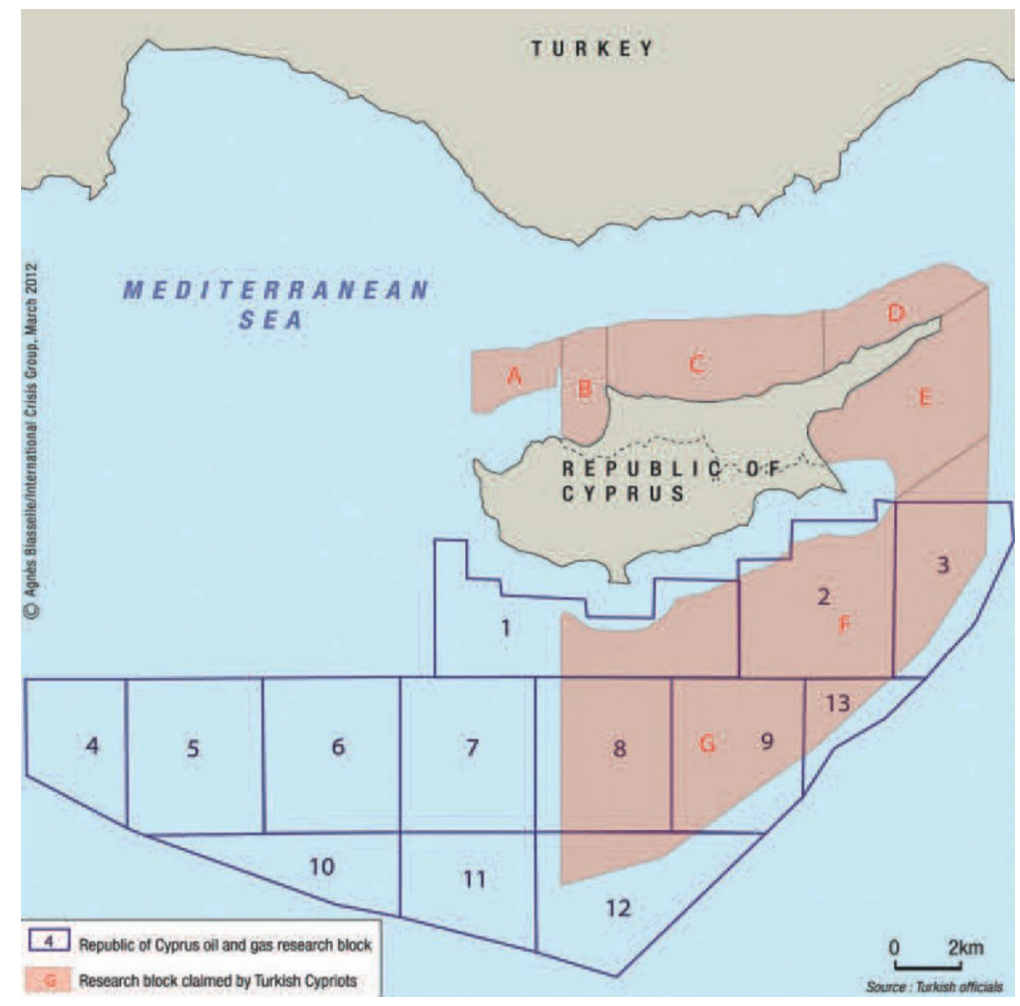

\section{CARTE 1. LES ZEE DE LA RÉPUBLIQUE DE CHYPRE ET REVENDIQUÉES PAR LA RTCN}

De 1 à 13, les blocs délimités par la République de Chypre. De A à G, les zones délimitées dans l'accord RTCN/Turquie.

Source: Faustmann et al., 2012, p. 27.

La Turquie en effet refuse de reconnaître les limites fixées par la République de Chypre et de négocier avec elle, car elle juge que cet État n'est pas un interlocuteur valable puisqu'il ne représente de facto que les Chypriotes grecs; protectrice des droits des Chypriotes turcs, seul État à avoir reconnu l'existence de la République turque de Chypre Nord (RTCN) proclamée unilatéralement en 1983, elle juge qu'aucune recherche ne peut avoir lieu avant le règlement de la «question chypriote ». Pour riposter aux premières recherches de la République de Chypre, elle signe à New York un accord avec la RTCN, en septembre 2011, qui fixe les limites entre les deux pays, la ZEE demandée par la RTCN et un accord entre la compagnie pétrolière turque TPAO et la RTCN fixe les zones de recherches qui 
lui sont allouées proches des côtes chypriotes, divisées en sept blocs, les uns situés au nord de l'île, les autres à l'est et au sud-est recoupant les blocs 1, 2, 3, 8, 9 et 13 définis par la République de Chypre.

À l'ouest de l'île, l'affaire se complique encore en raison du différend grécoturc. Depuis 1973 (et des recherches pétrolières en Égée) la Turquie refuse à la Grèce le droit d'étendre ses eaux territoriales en Égée de 6 à $12 \mathrm{MN}$, car cela donnerait aux Grecs près de $72 \%$ de la surface maritime et «l'étoufferait »; elle a même déclaré en 1995 que toute mesure grecque en ce sens serait à ses yeux un casus belli. Dans ce secteur en particulier, la question concerne l'île grecque de Castellorizo, à $130 \mathrm{~km}$ à l'est de Rhodes - et à un seul mille des côtes turques - et l'île de Strongyli, $9 \mathrm{~km}$ plus à l'est. Si on attribue à ces deux îles ZEE et plateau continental, les eaux grecques rejoignent la ZEE chypriote, ce qui renforce le complexe d'encerclement des Turcs, mais facilite politiquement l'exportation du gaz vers l'Europe. La Turquie soutient que Castellorizo n'est qu'une enclave sur le plateau continental turc et, à ce titre, ne justifie pas une ZEE particulière ${ }^{7}$. De ce fait, elle pourrait appliquer une ZEE de $200 \mathrm{MN}$ (ce qu'elle fait en mer Noire), et alors revendiquer une partie des blocs 1, 4, 5,6 et 7 définis par la République de Chypre. 


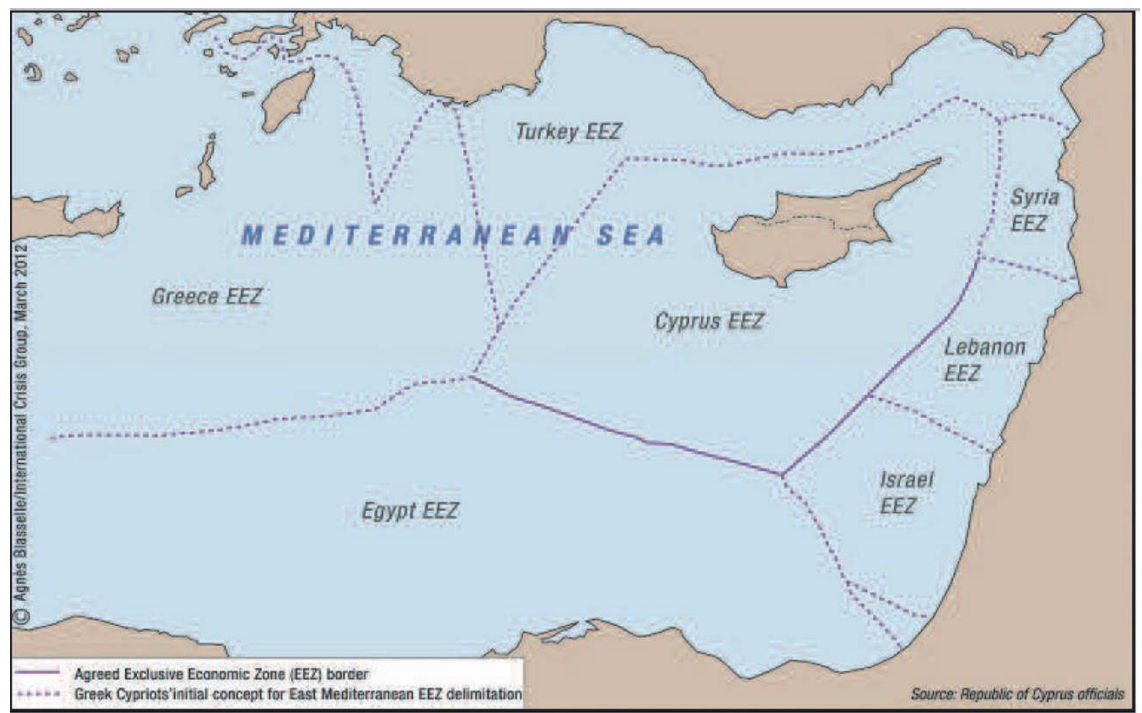

CARTE 2. LES ZEE (EEZ EN ANGLAIS) SELON LA GRÈCE ET LA RÉPUBLIQUE DE CHYPRE

Castellorizo se trouve entre Rhodes et Chypre, là où les pointillés frôlent la côte turque. Source: FAustmann et al., 2012, p. 29.

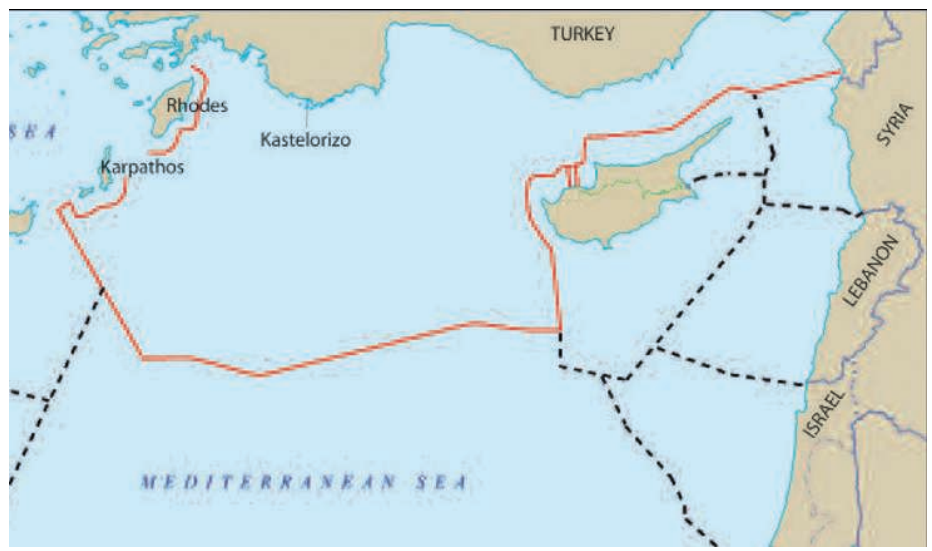

CARTE 3. LES ZEE DE LA GRÈCE, DE LA TURQUIE, DE LA RÉPUBLIQUE DE CHYPRE ET DE LA RTCN SELON LA VERSION TURQUE

Source: GÜREL et al., 2013, p. 29. 


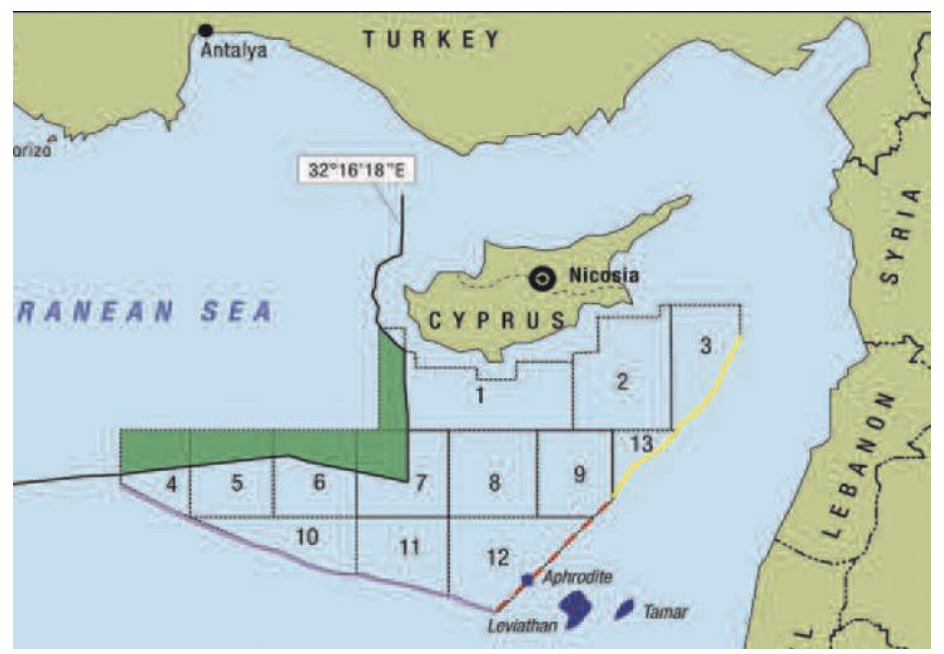

CARTE 4. LES «BLOCS » DÉFINIS PAR CHYPRE ET REVENDIQUÉS PAR LA TURQUIE

Source: FAustmann et al., 2012, p. 26.

Même si les puissances étrangères, ne reconnaissant pas la RTCN, acceptent la ZEE fixée par Chypre et semblent désavouer la Turquie, celle-ci n'en reste pas moins un obstacle pour les compagnies qui souhaitent des garanties à long terme.

\section{L'obstacle turc}

Dès 2003, la Turquie refuse à Chypre le droit de définir une ZEE; en 2007, elle lui dénie le droit de lancer des recherches. Elle développe ses arguments inchangés à chaque nouvelle campagne de recherche de Chypre en utilisant une rhétorique menaçante, et, à chaque fois, la Grèce se présente comme protectrice de la République de Chypre ${ }^{8}$, ce qui relance la querelle gréco-turque en Égée. Le gouvernement chypriote affirme les droits du seul État chypriote reconnu, précise régulièrement depuis 2006 qu'il est prêt à faire participer tous les Chypriotes aux bénéfices éventuels et en appelle à la protection de l'Union européenne, à l'ONU et aux États-Unis quand la tension paraît dangereuse.

La Turquie a d'abord tenté d'isoler Chypre dans sa définition de la ZEE en intervenant successivement auprès de l'Égypte et d' Israël sans succès; voyant plus

8. La République de Chypre, lors de sa création, a reçu trois puissances protectrices, la Grande-Bretagne, la Grèce et la Turquie. 
loin, en 2011, elle prévient l'Égypte qu'elle n'acceptera aucun accord de ZEE entre elle et la Grèce. Elle s'adresse aussi directement au gouvernement chypriote, ainsi en 2012, lors du deuxième appel d'offres, en l'avertissant qu'elle ne permettra aucune recherche dans les zones qu'elle estime siennes et «prendra les mesures nécessaires ${ }^{9}{ }^{\prime}$, et qu'elle est prête à protéger les droits de la RTCN dans les zones qu'elle considère comme siennes et où la TPAO (Turkish Petroleum) a reçu une licence de recherche.

Elle cherche surtout à effrayer les compagnies étrangères qui craignent pour les investissements importants que les recherches entraînent. Ainsi, en 2012, elle fait savoir à l'ENI qu'elle pourrait reconsidérer sa position face à ses investissements en Turquie. Mais surtout, elle envoie des navires de guerre menaçants à proximité des lieux de forage. L'année 2018, réunit tous les éléments de l'affrontement en raison de trois éléments nouveaux : l'échec des négociations sur la réunification de Chypre dans l'été 2017, l'annonce de la découverte du prometteur gisement Calypso au début de janvier 2018, l'arrivée du navire de recherches affrété par l'ENI pour des recherches sur le bloc 3 (dans la ZEE revendiquée par la Turquie). On trouve donc:

- le renouveau de tension entre la Grèce et la Turquie qui accompagne en général ces phases de conflit;

Depuis le refus grec de renvoyer en Turquie huit officiers en fuite après le coup d'État de l'été 2016, les incidents entre les flottes militaires des deux pays se sont multipliés - trois fois à proximité de l'îlot d'Imia (déjà au centre d'un grave épisode en 1996) -, chacun accusant l'autre d'être le responsable. Le 12 février 2018, un bateau de patrouille turc a éperonné un navire de la garde côtière grecque devant Imia.

- Le gonflement rhétorique;

Tandis que le 18 février 2018, le Président turc Erdoğan rappelle que la Turquie a autant de droits à intervenir à Afrin en Syrie qu'en mer Égée ou à Chypre, le lendemain, le Premier ministre grec parle des violations provocatrices des Turcs dans les eaux territoriales et l'espace aérien grec en Égée, et le 24 février 2018, le Premier ministre turc Binali Yıldırım déclare que: «la marine militaire turque a la puissance de faire disparaittre tout danger en Égée et en Méditerranée, quel que soit le danger et la conjoncture. » L'Union européenne, fidèle à elle-même, invite

9. GÜRel, 2013, p. 61-74; FaUstmann, 2012, p. 9-30. 
la Turquie à éviter les menaces et à s'abstenir de toute action qui pourrait nuire à des relations de bon voisinage.

- La menace sur les compagnies.

Le 22 février 2018, le navire SAIPEM 12000 de l'ENI, immobilisé depuis deux semaines par des frégates turques, décide de quitter les lieux pour le Maroc et assure... qu'il reviendra. Une première. Au même moment, Exxon-Mobil envoie lui aussi un navire d'exploration vers le bloc qui lui a été accordé en assurant qu'il ne cédera pas.

Depuis lors, les relations entre la Grèce et la Turquie se tendent chaque jour, le président Erdoğan ne recule pas d'un pas dans ses déclarations nationalistes, assurant que la Turquie est prête à réitérer son intervention de 1971 pour défendre les intérêts des Chypriotes turcs ${ }^{10}$. La situation, dangereuse en 2018, l'est encore davantage au printemps 2019; au début du mois de mai, la Turquie envoie deux, puis trois de ses navires de forages, dans la zone qu'elle revendique à l'ouest de l'île, un quatrième est sur le point de les rejoindre en août 2019. Immédiatement la République de Chypre, soutenue par le gouvernement grec, en appelle à l'Union européenne et aux Nations unies, le 29 mai 2019, L'Opinion titre «Chypre au cœur des grandes tensions gazières en Méditerranée orientale ». L'Union européenne adresse effectivement des remarques acerbes à la Turquie puis refuse de verser une partie des fonds promis pour faciliter son intégration européenne ${ }^{11}$, R. T. Erdoğan riposte par des menaces voilées sur les accords conclus à propos des migrants qu'il prend en charge, les États-Unis, mécontents de voir la Turquie acheter des systèmes antimissiles $S 400$ à la Russie, critiquent également ces initiatives. En juillet 2019, pour améliorer son image de protecteur de la RTCN, la Turquie pousse le président de Chypre Nord, Mustafa Akıncı, à proposer à la République de Chypre une coopération dans la prospection et l'exploitation, ce que Nicosie ne peut que refuser pour ne pas reconnaître de facto la RTCN comme État ${ }^{12}$. Quelle que soit l'issue de l'affaire, on comprend bien que les tensions Chypre/Turquie sont un facteur très défavorable pour l'exploitation des ressources off-shore. Néanmoins, elles ne sont pas le seul, la rentabilité des exportations en est un autre, aussi essentiel.

10. Kathimerini, 20/7/2019.

11. Le Temps, 21/7/2019.

12. Challenges, 13/7/2019. 


\section{Comment mettre en valeur?}

Les projets et techniques possibles de mise en valeur et d'exportation du gaz sont aussi fonction des critères de rentabilité. Les prix bas et l'abondance du gaz russe sont un handicap pour des exportations; toutes les techniques envisagées actuellement conduiraient en Europe du gaz à des prix bien supérieurs à ceux des approvisionnements d'aujourd'hui, et le choix dépend aussi des réserves réellement exploitables, encore mal connues.

L'Égypte est bien placée, le gaz israélien de Tamar et le gaz de Zohr peut, par gazoducs sous-marins, rejoindre les installations de gaz naturel liquéfié (GNL) d'Idku et de Damiette, contribuer à assouvir la très forte demande locale et être exporté. En août 2016, Chypre et l'Égypte ont signé un accord pour acheminer le gaz de Calypso en Égypte où il serait liquéfié et exporté. Les arrivées de gaz israélien sont prévues pour $2019^{13}$, un autre accord prévoit d'acheminer vers l'Égypte, par conduite sous-marine, le gaz d'Aphrodite en 2022.

Comment et sous quelle forme exporter les ressources chypriotes ${ }^{14}$ ? Les différentes solutions envisagées reposent sur un triangle Israël-Chypre-Grèce qui s'est construit par de nombreux accords et visites officielles réciproques et d'accords, y compris militaires, entre la Grèce et Israël à partir de 2010 et Israël et Chypre à partir de 2012. Quelles sont les solutions possibles?

Les autorités chypriotes ont envisagé en 2012 la construction d'une station de fabrication de GNL (gaz naturel liquéfié) dans le port industriel de Vasilikos au sud de l'île. Le gaz arriverait par oléoducs de Leviathan et d'Aphrodite (qui, avec des ressources estimées à 127 milliards de mètres cubes, ne justifie pas à elle seule une telle implantation). L'exportation est ensuite facile, mais le coût de l'équipement est élevé et suppose qu' Israël soit prêt à exporter ${ }^{15}$.

Certaines études citent les possibilités techniques récentes, le FCNG (gaz naturel compressé flottant) et le FLNG (gaz naturel liquéfié flottant), mais aucune étude n'a été faite pour les adapter à la Méditerranée ${ }^{16}$.

La solution souvent citée comme la moins chère et la plus rapide à réaliser est celle d'un gazoduc passant par Chypre et gagnant le Sud de la Turquie (qui a besoin d'énergie) où il rejoindrait les réseaux locaux et européens par Mersin-Adana; malgré ses avantages, ce qui précède montre clairement que

13. VOA Afrique, $27 / 9 / 2018$.

14. GÜREL et al. 2013, p. 75-88. Voir les solutions techniques dans la partie "The energy market and economic context".

15. Erpic, 2017 ; LAKES, 2012, p. 89-95.

16. Enotiades \& Pitatzis, 2017. 
c'est diplomatiquement irréalisable pour l'instant, et inacceptable par l'opinion publique au Sud de l'île.

L'attention s'est donc portée sur des solutions court-circuitant la Turquie que l'Union européenne patronne dans le but de réduire sa dépendance par rapport au gaz russe. Un gazoduc sous-marin, dit Eastmed, passant par la Crète (où des forages de recherche ont lieu) rejoindrait la Grèce et le marché européen, par l'Italie, soit $1150 \mathrm{~km}$ (près de $2000 \mathrm{~km}$ avec la liaison italienne), qui aurait, à l'horizon 2025, une capacité de transport entre 12 et 16 milliards $\mathrm{m}^{3}$ par an ${ }^{17}$, un exploit technique réalisable, mais extrêmement coûteux vu les grandes profondeurs par endroits et les risques sismiques, qui demande donc un approvisionnement sûr pendant des décennies ${ }^{18}$. Ce projet, en germe depuis 2013, dont l'étude technique est financée depuis 2015 par l'Union européenne, a été annoncé en janvier 2016 lors d'un sommet israélo-gréco-chypriote; la Commission européenne l'a inscrit dans la liste des 195 projets d'infrastructures énergétiques dits « d'intérêt commun », mais il reste au stade des études.

On pense parallèlement à transformer du gaz en électricité sur l'île de Chypre et à un câble sous-marin, partant d'Israël, qui la transporterait en Europe via la Crète et la Grèce, un long trajet, mais comparable à ceux qui relient déjà la Norvège aux Pays-Bas ou la Sardaigne à l'Italie. Ce projet fait partie également des projets déclarés d'intérêt commun en 2017 parmi les autoroutes de l'électricité, c'est l'Euroasia interconnector. Parallèlement au câble électrique, un câble sous-marin de fibre optique, aux capacités gigantesques, Quantum Cable, long de $7700 \mathrm{~km}$, doit relier le Proche-Orient (et l'Asie) à l'Europe jusqu'à Bilbao en passant par Chypre et la Crète; il devrait rallier la Grèce en $2022^{19}$.

\section{Conclusion}

Nicosie, se présentant comme un partenaire plus sûr que la Turquie, souhaite devenir une plate-forme régionale dans le domaine des hydrocarbures avec l'installation d'usines de liquéfaction de gaz et d'oléoducs reliant Israël et le Liban; on parle dans ce secteur de plusieurs milliards de mètres cubes avec de possibles gisements de pétrole en dessous des couches de gaz! Les dernières décisions européennes feraient même de Chypre et de la Crète (par les sites de Kofinou et d'Héracleion) un centre (bub) mondial d'échanges, renforçant la

17. Parlement Européen, 2018.

18. MARgheri, 2017.

19. Quantum Cable. 
situation politique de la République de Chypre. Mais leur point commun est un coût très élevé qui fournirait du gaz à un prix bien supérieur au coût actuel ${ }^{20}$. La question reste donc en suspens. De ce fait, elle remet en question l'enthousiasme qu'avaient provoqué à Chypre les premières découvertes. On espérait alors que les ressources des hydrocarbures pourraient aider à résoudre les problèmes financiers que provoquerait une éventuelle réunification de l'île, que l'intérêt commun pourrait pousser les Chypriotes grecs et turcs, et même la Turquie, à plus de souplesse dans les négociations sur l'avenir de l'île, d'autant plus que les dirigeants se déclaraient décidés à partager les bénéfices avec tous les habitants ${ }^{21}$. Aujourd'hui, devant les blocages et les menaces et la déclaration répétée selon laquelle il ne peut y avoir d'exploitation avant une réunification de l'île, l'avenir est plus sombre. Il est surtout plus complexe dans la mesure où il repose sur les relations diplomatiques fragiles entre les deux parties de Chypre, entre Chypre, la Grèce et la Turquie, entre la Turquie et Israël, entre Israël et ses voisins et... sur les réactions des compagnies pétrolières.

\section{Bibliographie}

\section{Monographies}

Ellinas Charles, Roberts John \& Tzimitras Harry, 2016, Hydrocarbon developments in the Eastern Mediterranean, the case for pragmatism, Atlantic Council, Global Energy Center and Dinu Patriciu Eurasia Center.

Faustmann Hubert, Gürel Ayla, Reichberg Gregory M., 2012, Kvлgıaxoí

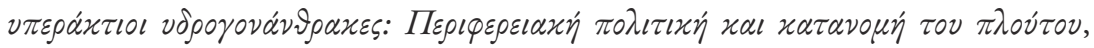
[hydrocarbures off-shore chypriotes: politique périphérique et répartition de la richesse], PRIO Cyprus-Friedrich Ebert Stiftung, nº 1, Nicosia.

Gürel Ayla, Mullen Fiona \& Tzimitras Harry, 2013, The Cyprus Hydrocarbons Issue: Context, Positions and future scenarios, PRIO Cyprus, report 1, Nicosia.

Tzimitras Harry, 2014, Hydrocarbons in the Eastern Mediterranean: agent of conflict or platform for cooperation, in The Peace potential of Hydrocarbons resources, a scoping study, PRIO Cyprus, Nicosia.

20. Cyprus Mail, 30/1/2018.

21. GÜrel, 2016, p. 117-137; ICG, 2012; Tzimitras, 2014; Ellinas, 2016. 


\section{Articles et participations à des ouvrages}

Ellinas Charles, 2016, "Eastern Mediterranean gas developments", in Gürel Ayla, Tzimitras Harry \& Faustmann Hubert, Global Energy Debates and the Eastern Mediterranean, PRIO Cyprus Centre-Friedrich Ebert Stiftung-Atlantic Council, PRIO Oslo.

Gürel Ayla, 2016, "Eastern Mediterranean Gas: source of prosperity for the Region?”, in Colombo Silvia, El Harrak Mohamed \& Sartori Nicolò, The Future of Natural Gas, Markets and Politics, Istituto Affari internationali, European Energy Review, Leiden, Pays-Bas, pp. 117-137.

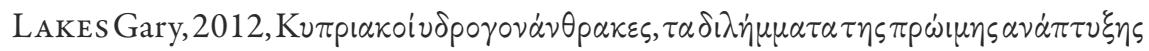
[Hydrocarbures chypriotes, les dilemnes d'un premier développement] in

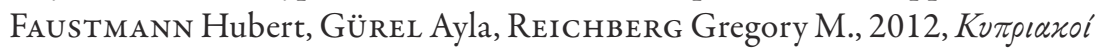

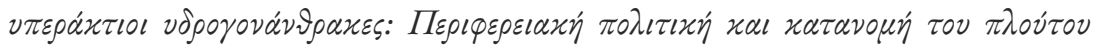
[hydrocarbures off-shore chypriotes: politique périphérique et répartition de la richesse], PRIO Cyprus-Friedrich Ebert Stiftung, nº 1, Nicosia. p. 89-95.

\section{Presse}

Boursedirect, 8/2/2018, «ENI et Total découvrent d'importantes réserves sousmarines de gaz à Chypre », URL: https://www.boursedirect.fr/fr/actualites/ categorie/economie/eni-et-total-decouvrent-d-importantes-reserves-sousmarines-de-gaz-a-chypre-afp-7a422cf72f0b993440ab1e06b310fb7652 7 fe067 (consulté le 29/11/2019).

Challenges, 13/7/2019, «Chypre: la partie turque propose à Nicosie de coopérer à la prospection gazière », URL: https://www.challenges.fr/monde/ chypre-la-partie-turque-propose-a-nicosie-de-cooperer-a-la-prospectiongaziere_664058 (consulté le 29/11/2019).

Cyprus Mail, 30/1/2018, "Cyprus : Price, not politics, stymying East Med gas", URL: http://www.hellenicshippingnews.com/cyprus-price-not-politicsstymying-east-med-gas (consulté le 29/11/2019).

Enotiades Christos \& Pitatzis Athanasios, 2017, "FCNG: A Solution to Unlock the Full Potential of East Med Hydrocarbons “, URL: https://www. 
linkedin.com/pulse/fcng-solution-unlock-full-potential-east-med-thanospitatzis (consulté le 29/11/2019).

Erpic, 2017, "Eastern Mediterranean gas fields and a new energy corridor to Europe", Erpic.org, URL: https://erpic.org/wp-content/uploads/2017/02/ erpic-eastern-mediterranean-gas-fields-and-a-new-energy-corridor-toeurope-0912.pdf (consulté le 29/11/2019).

Exxon-Mobil, 1/3/2019, URL: http://www.petrole-et-gaz.fr/exxonmobilannonce-une-importante-decouverte-de-gaz-au-large-de-chypre-13268/ (consulté le 29/11/2019).

Gotev Georgi, 2018, «L'Union fait pression sur la Turquie sur la zone économique chypriote », Euractiv, URL: https://www.euractiv.fr/section/ politique/news/eu-turkey-varna-summit-becomes-conditional (consulté le $29 / 11 / 2019$ ).

ICG, 2012, "Aphrodite gift's: Can Cypriot gas power a new dialogue?", International Crisis Group, Europe Report, no 216, URL: https://www. crisisgroup.org/europe-central-asia/westerneuropemediterranean/cyprus/ Aphrodite (consulté le 29/11/2019).

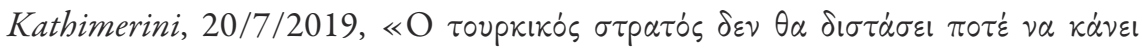

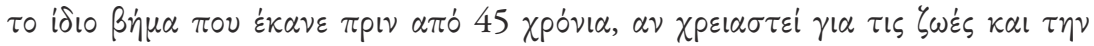

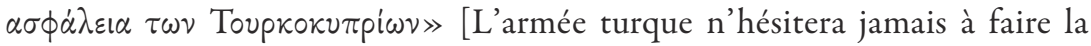
même chose qu'il y a 45 ans s'il le faut, pour les vies et la sécurité des Turcs de Chypre], URL: https://www.kathimerini.com.cy/gr/politiki/erntogan-gia45-xronia-apo-tin-toyrkiki-eisboli-ean-xreiastei-tha-epanalaboyme-to-idiobima (consulté le 29/11/2019).

Le Monde de l'énergie, 12/12/2017 «Italie, Grèce, Chypre et Israël ouvrent la voie au pipeline méditerranéen Eastmed », URL: http://www.lemondedelenergie. com/pipeline-eastmed/2017/12/12 (consulté le 29/11/2019).

Le Temps, 21/7/2019, «Les promesses du gaz off-shore ravivent les tensions entre Ankara et Chypre», URL: https://www.info-grece.com/ actualite/2019/07/21/les-promesses-du-gaz-offshore-ravivent-les-tensionsentre-ankara-et-chypre (consulté le 29/11/2019). 
MARGHeRI Marco, 2017, The East Med Pipeline project: a project of common interest for the diversification of gas supplies to Europe, EDISON-EDF Group, URL: http://www.eurogas.org/uploads/media/EDISON_MargheriEastMed_ EP_8.03.2017.pdf (consulté le 29/11/2019).

Parlement européen, http://www.europarl.europa.eu/meetdocs/2014_2019 /plmrep/COMMITTEES/ITRE/DV/2018/01-22/COM_ADL201 707834ANN_FR.pdf (consulté le 29/11/2019).

Pipes Daniel, 2012, «Kastellorizo véritable poudrière méditerranéenne » in National Review online, URL: http://fr.danielpipes.org/10646/kastellorizo (consulté le 29/11/2019).

Quantum Cable, “About us”, URL: http://www.euroasia-interconnector.com/ about-us/quantum-cable (consulté le 29/11/2019).

Reuters, 26/3/2015, "ENI-KOGAS finds no exploitable hydrocarbons off Cyprus”, URL: http://reut.rs/1GsPvv5 (consulté le 29/11/2019).

STUder Élisabeth, 2017, Chypre: nouveaux contrats d'exploration pour Total, Exxon, Eni et Qatar Petroleum, URL: http://www.leblogfinance. com/2017/03/chypre-nouveaux-contrats-dexploration-pour-total-exxoneni-et-qatar-petroleum.html (consulté le 29/11/2019).

USGC (United States Geological Surveys), 2010, Assessment of Undiscovered Oil and Gas Resources of the Levant Basin Province Eastern Mediterranean, Fact Sheet 2010-3014, US Department of the Interior.

VOA Afrique, 27/9/2018, «Du gaz israélien exporté vers l'Égypte dès début 2019», https://www.voaafrique.com/a/du-gaz-isra\%C3\%A9lienexport\%C3\%A9-vers-l-egypte-d\%C3\%A8s-d\%C3\%A9but-2019/4589487. html (consulté le 29/11/2019).

Résumé: la découverte depuis une dizaine d'années de ressources en gaz naturel très importantes dans le bassin du Levant, entre Chypre, la côte moyen-orientale et l'Égypte, a fait naître des espoirs importants dans les États riverains, Chypre y compris. Mais ils firent place très vite aux difficultés : à qui appartiennent les ressources du plateau continental ? Et si ce problème est résolu, 
comment transporter ce gaz jusqu'aux consommateurs et sous quelle forme? À Chypre, ces questions sont devenues sources d'incertitudes étant donné la situation politique actuelle de l'île, elle-même imbriquée dans le différend grécoturc. Aujourd'hui, elles sont à l'origine de graves tensions en Méditerranée orientale. C'est là le sujet de cet article.

Mots-clefs : Chypre, gaz naturel, ZEE, différend gréco-turc, bassin du Levant, $\mathrm{XXI}^{\mathrm{e}}$ siècle, Eastmed

Abstract: the discovery over the past decade of significant natural gas resources in the Levantine basin between Cyprus, the Middle East Coast and Egypt has raised important hopes in riparian States, including Cyprus. But they gave way very quickly to difficulties: who owns the resources of the continental shelf? And if this problem is solved, how to transport this gas to consumers and in what form? In Cyprus, these issues have become major problems, given the current political situation of the island, which is itself embedded in the Greco-Turkish dispute. Today, they cause serious tensions in the eastern Mediterranean. This is the subject of this article.

Keywords: Cyprus, natural gas, EEZ, Greco-Turkish dispute, Levantine Basin, $21^{\text {st }}$ century, Eastmed

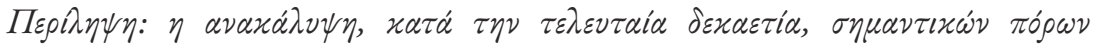

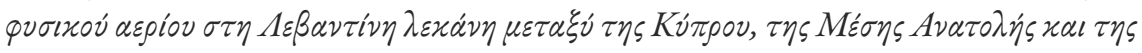

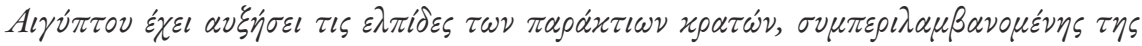

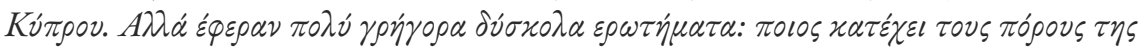

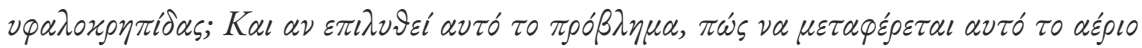

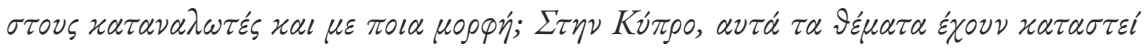

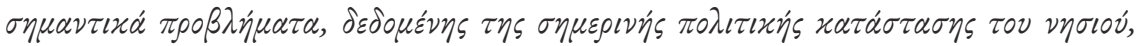

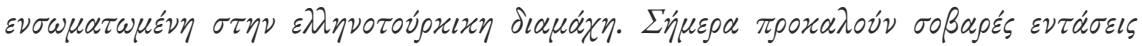

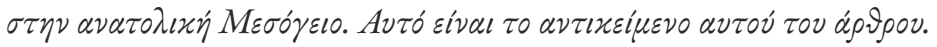

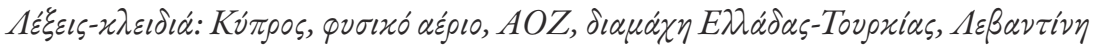
$\lambda \varepsilon x a ́ v \eta, 210 \varsigma \alpha \iota \omega ́ v \alpha \varsigma$, Eastmed

Anahtar kelimeler: Kıbrıs, doğal gaz, EEZ, Türkiye Greco anlaşmazliğı, Levanten havzasi, 21. yüzyıl, Eastmed

Клучни зборови: Кипар, природен гас, EЕ3, турски грчки спор, левантински бaceu, 21 век, Eastmed 